\title{
Levo-Transposition of the Great Arteries
}

National Cancer Institute

\section{Source}

National Cancer Institute. Levo-Transposition of the Great Arteries. NCI Thesaurus. Code C98902.

An acyanotic congenital cardiovascular abnormality characterized by the transposition of the aorta and the pulmonary artery. 